\title{
Origem e distribuição anatômica do nervo isquiático de mão-pelada ${ }^{1}$
}

\begin{abstract}
Kleber F. Pereira ${ }^{2 *}$, Juliana F.F.e S. Paranaiba ${ }^{2}$, Carla Helrigle ${ }^{2}$ e Eugênio G. de Araújo ${ }^{3}$
ABSTRACT.- Pereira K.F., Paranaiba J.F.F.S., Helrigle C. \& Araújo E.G. 2011. [Origin and anatomical distribution of ischiatic nerve in the crab-eating raccoon (Procyon cancrivorus).] Origem e distribuição anatômica do nervo isquiático de mão-pelada (Procyon cancrivorus). Pesquisa Veterinária Brasileira 31(Supl.1):74-78. Laboratório de Anatomia Humana e Comparativa, Curso de Ciências Biológicas, Universidade Federal de Goiás, Campus Jataí, BR 364 Km 192, Setor Parque Industrial, Jataí, GO 75800-000, Brazil. E-mail: kpereiraufg@gmail.com

The ischiatic nerve is the largest nerve in the body, belongs both to the sacral and lumbosacral plexus in carnivores, continuing until the distal hind limb, and receives fibers from the ventral branch of the sixth and seventh lumbar nerves and the first sacral nerve. We aim to describe the distribution of the sciatic nerve in raccoon (Procyon cancrivorus) and compare with data of literary domestic and wild animals. The animals are coming to collect on highways (killed by accident) and subjected to fixation in aqueous solution, $10 \%$ formaldehyde. The dissection and photographic documentation (Sony $\alpha 200$ camera, 10.2mpx) shows the distribution of the sciatic nerve. The ischiatic nerve of raccoon innervates the hind limb passing between the middle and deep gluteal muscles, giving off branches to the muscles of the buttock and thigh, respectively, for the gluteus medius, gluteus biceps, semimembranosus, semitendinosus, biceps femoris, twins, quadratus femoris and adductor magnus, sending the lateral cutaneous nerve of the thickness and flow to supply the skin on the lateral surface of the leg and tail, respectively. Near the middle of the thigh nerve bifurcates into the tibial and peroneal nerve. The anatomical knowledge of the pattern of nervous raccoon (Procyon cancrivorus) is of fundamental importance in research to refer to the distribution of the ischiatic nerve, and show no disagreement with the corresponding data in the literature of domestic carnivores.
\end{abstract}

INDEX TERMS: Anatomy, ischiatic nerve, Procyon cancrivorus.

RESUMO.- O nervo isquiático é considerado o maior nervo do corpo, pertence tanto ao plexo sacral quanto ao lombossacral em carnívoros, continuando até a extremidade distal do membro pélvico, recebe fibras dos ramos ventrais do sexto e sétimo nervos lombares e do primeiro nervo sacral. O objetivo do presente estudo é descrever a distribuição do nervo isquiático em mão-pelada (Procyon cancrivorus) e comparar com dados literários de animais domésticos e silvestres. Os animais são procedentes de coleta em ro-

\footnotetext{
${ }^{1}$ Recebido em 8 de setembro de 2011.

Aceito para publicação em 29 de novembro de 2011.

${ }^{2}$ Curso de Ciências Biológicas, Universidade Federal de Goiás (UFG), Campus Jataí, Unidade Jatobá, BR 364 Km 192, Setor Parque Industrial, Jataí, GO 75800-000, Brasil. *Autor para correspondência: kpereiraufg@ gmail.com

${ }^{3}$ Escola de Veterinária e Zootecnia, UFG, Campus Samambaia, Cx. Postal 131, Goiânia, GO 74001-970.
}

dovias, entre as cidades de Goiânia e Jataí, principalmente na BR 364 ou BR 060. (mortos por acidente) e fixados em solução aquosa, a $10 \%$ de formaldeído. Doados ao Museu de Anatomia Humana e Comparada da UFG (Universidade Federal de Goiás, Campus de Jataí, Proc.CAJ-287/2008). As dissecações e documentação fotográfica permitiram observar a distribuição do nervo isquiático. 0 nervo isquiático de mão-pelada inerva o membro pélvico passando entre os músculos glúteo médio e profundo, emitindo ramos para a musculatura da região glútea e da coxa, respectivamente, para os músculos glúteo médio, glúteo bíceps, semimembranáceo, semitendíneo, bíceps femoral, gêmeos, quadrado femoral e adutor magno, emitindo nervo cutâneo lateral e caudal da sura para suprir a pele na superfície lateral e caudal da perna, respectivamente. Próximo ao meio da coxa bifurca-se em nervo tibial e nervo fibular comum. 0 conhecimento anatômico da origem e distribuição do nervo is- 
quiático em mão pelada quando comparado com animais domésticos, silvestres e de fazenda, mostram um padrão de inervação semelhante entre os espécimes.

TERMOS DE INDEXAÇÃO: Anatomia, nervo isquiático, Procyon cancrivorus.

\section{INTRODUÇÃO}

O mão-pelada é um mamífero carnívoro da família Procyonidae. Sua distribuição geográfica inclui as Americas Central e do Sul, do leste da Costa Rica e do Panamá, até o Uruguai e o norte da Argetina, e no Brasil se distribui por todo território (Carvalho 1983, Câmara \& Murta 2003, Cubas et al. 2006). De coloração cinza, quase preta, possui uma máscara negra ao redor dos olhos e a cauda com anéis. Vive em áreas de florestas, principalmente perto de rios e lagos. É um animal noturno que se alimenta de frutos, insetos, caranguejos, peixes e outros pequenos animais (Emmons \& Feer 1997, Nowak 1999, Júnior \& Luz 2008). Possui o tato bem desenvolvido e agilidade manual o que o permite procurar por peixes e outros organismos aquáticos em água rasa ou lodo, geralmente lavando-os antes de ingeri-los (Silva 1994, Miranda 2003, Reis et al. 2006).

A anatomia tem como base o estudo macro e microscópico do corpo, que subsidiam a análise descritiva de uma espécie e/ou para comparação entre espécies que apresentem semelhanças morfológicas. Esta pesquisa justifica-se devido ao escasso número de trabalhos publicados na literatura brasileira e mundial acerca da espécie (Pereira et al. 2010), principalmente no que se refere à descrição da origem e distribuição dos nervos periféricos. De acordo com Aversi-Ferreira et al. (2005), o método de dissecação é o mais direto para a observação das estruturas corpóreas. As preparações anatômicas possibilitam a exposição dos músculos e nervos, sendo possível o acompanhamento de origens e distribuições.

O nervo isquiático é considerado o maior nervo do corpo, pertence tanto ao plexo sacral quanto ao lombossacral em carnívoros, continuando até a extremidade distal do membro pélvico, recebe fibras dos ramos ventrais do sexto e sétimo nervos lombares e do primeiro e segundo nervos sacrais (Schwarze \& Schröder 1970, Ellenberger \& Baum 1985, Konig \& Liebich 2004). Caudalmente, passa pela coxa entre o trocânter maior do fêmur e a tuberosidade isquiática, ao longo da superfície lateral do músculo semimembranáceo, emitindo ramificações para o músculo glúteo médio, glúteo bíceps, semimembranáceo, semitendíneo, bíceps femoral, gêmeos, quadrado femoral e adutores. Na região medial da coxa, o nervo isquiático dobra-se distalmente e se bifurca em nervo fibular e nervo tibial (Ghoshal 1986, Aversi-Ferreira 2011).

Sobre as descrições morfológicas do mão-pelada, foram desenvolvidas pesquisas relativas aos músculos do antebraço (Lima et al. 2010b), intestino (Lima et al. 2010a), perna (Pereira et al. 2010a) e coxa (Pereira et al. 2010b). 0 objetivo do presente estudo é descrever a origem e distribuição do nervo isquiático em mão-pelada, já que está entre as espécies de carnívoros brasileiros menos estudados (Morato et al. 2004), comparando com dados literários de animais domésticos, silvestres e de fazenda.
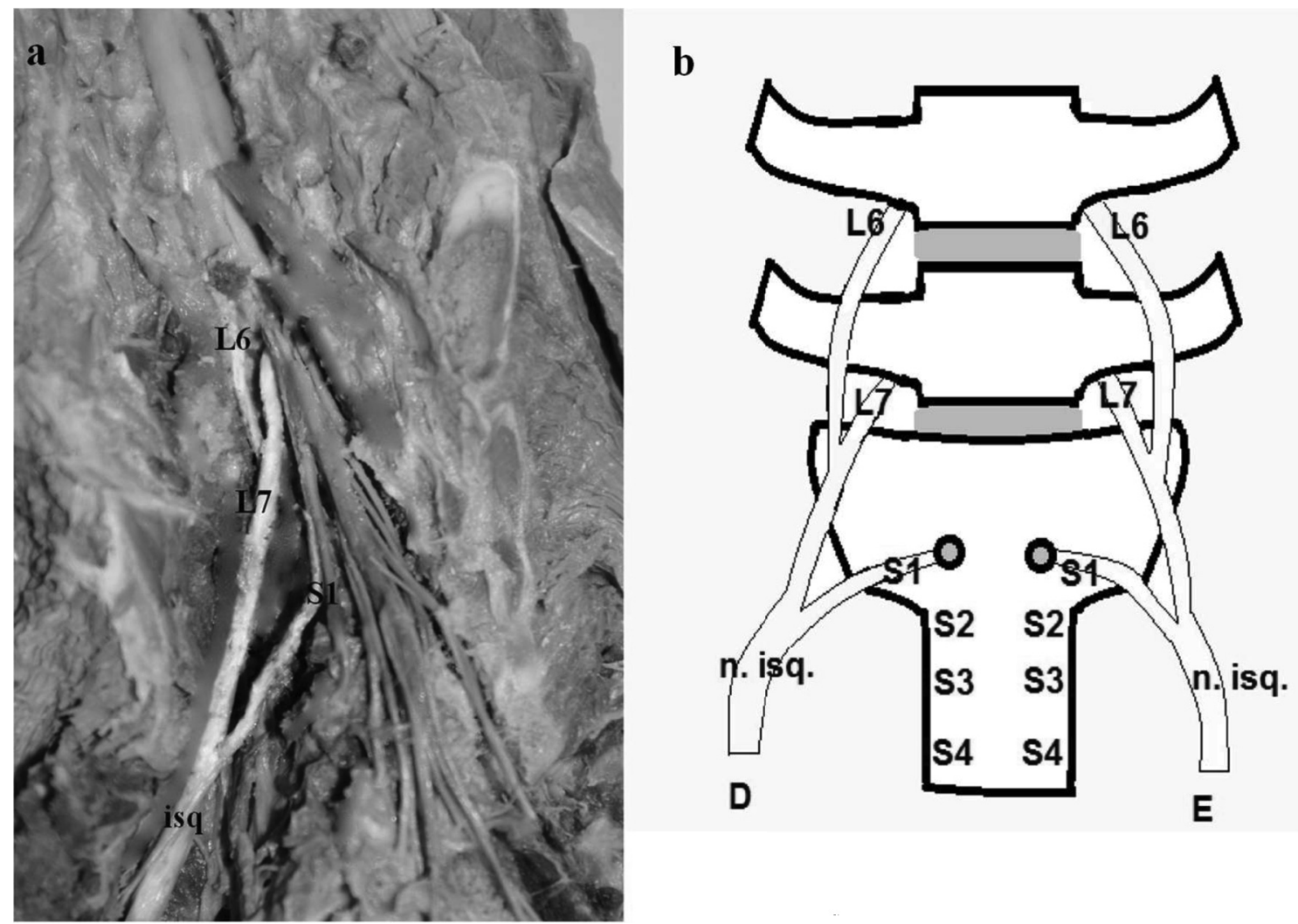

Fig.1. (a) Face dorsal do membro pélvico esquerdo e (b) desenho esquemático da região lombar e sacral da coluna vertebral, mostrando a origem do nervo isquiático (isq.) a partir dos ramos da L6, L7 e S1. 


\section{MATERIAL E MÉTODOS}

Este estudo baseou-se na análise descritiva do nervo isquiático em três exemplares de Procyon cancrivorus, machos, adultos, procedentes de coleta em rodovias (mortos por acidentes) cujos critérios obedeceram a Lei Vigente (Lei 1.153/95) e fixados em solução aquosa, a 10\% de formaldeído.

A descrição da distribuição do nervo isquiático foi realizada por meio do método de dissecação e posteriormente os resultados obtidos foram documentados com câmera fotográfica digital (Câmera Sony a200,10.2mpx) e por desenhos esquemáticos. Os dados obtidos foram comparados com dados da literatura do nervo isquiático de animais domésticos, como cão e gato, animais silvestres, como o preá, mocó e veado catingueiro e animais de fazenda, como ovinos, caprinos e bovinos, e foram adotadas as respectivas designações anatômicas, obedecendo ao International Commitee on Veterinary Gross Anatomical Nomenclature (2005).

\section{RESULTADOS}

O nervo isquiático de mão-pelada inerva o membro pélvico, origina-se no tronco lombossacral, nos ramos ventrais do sexto e sétimo nervos lombares e do primeiro nervo sacral (Fig.1). 0 nervo isquiático apresenta-se de forma semelhante no antímero direito e esquerdo nos três espécimes estudados (Fig.1b). Ele desce pela coxa entre o trocânter maior do fêmur e a tuberosidade isquiática, entre o músculo glúteo superficial, glúteo bíceps, glúteo profundo, bíceps femoral e abdutor caudal crural.
Em relação à distribuição do nervo isquiático, observamos cranialmente ramos suprindo os músculos glúteo bíceps e glúteo médio. Seguindo seu percurso caudalmente têm-se ramificação para os músculos bíceps femoral, semitendíneo, glúteo profundo, gêmeos, quadrado femoral, adutor magno e para o músculo semimembranáceo observam-se vários ramos distribuindo-se em todo o músculo. Na região lateral, o nervo isquiático emite o nervo cutâneo lateral da sura, que supre toda a superfície lateral da pele (Fig.2a e 2b).

Na região medial da coxa o isquiático se bifurca em nervo tibial (Fig.2b), suprindo os músculos caudais à tíbia e a fíbula e emite ramos para o joelho, e em nervo fibular comum que segue seu trajeto ramificando-se em nervo fibular superficial e profundo (Fig.2c), suprindo os músculos flexores do tarso e extensores dos dedos.

\section{DISCUSSÃO}

Devido à escassez de trabalhos científicos acerca da morfologia do mão-pelada, tem sido realizadas descrições anatômicas sobre outros sistemas orgânicos deste animal, como a anatomia dos músculos do antebraço e intestino (Lima et al. 2010), perna e coxa (Pereira et al. 2010), sendo que seus resultados indicam similaridades com as respectivas estruturas em carnívoros domésticos, como cães e gatos.
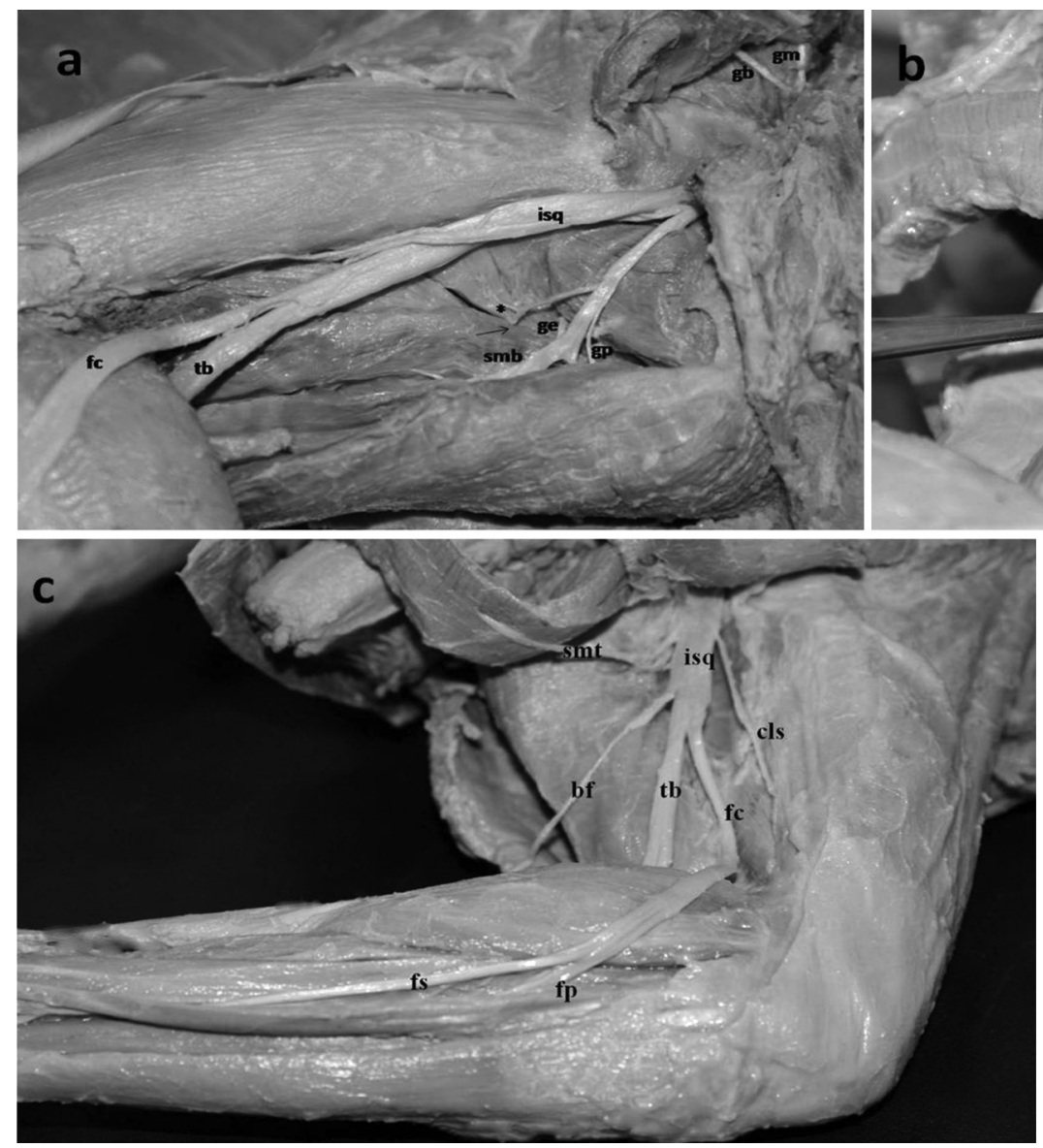

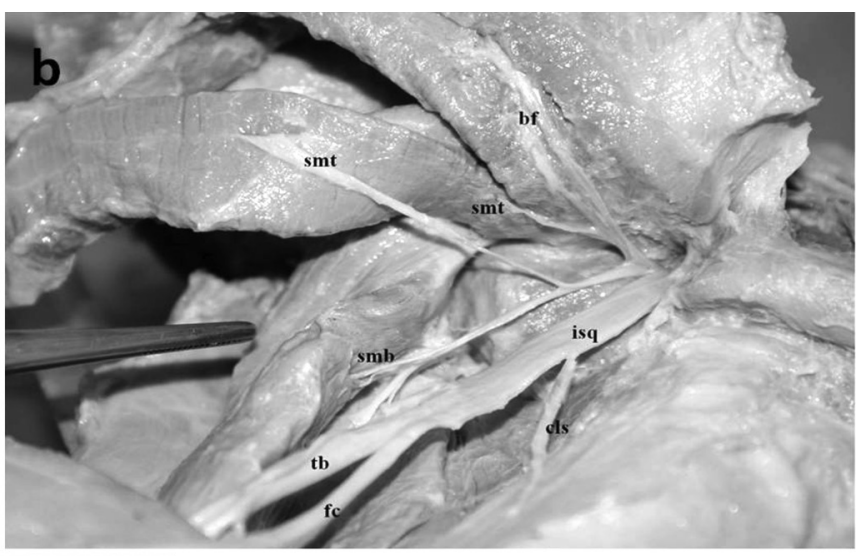

Fig.2. (a) Face dorsal do membro pélvico esquerdo, observam-se ramos do nervo isquiático (isq) para os músculos glúteo médio (gm), glúteo bíceps (gb), glúteo profundo (gp), gêmeos (ge), quadrado femoral (*), adutor magno (seta), semimembranáceo (smb), nervo fibular comum (fc) e nervo tibial (tb). (b) Membro pélvico direito, visibilizando o nervo isquiático (isq) e seus ramos para os músculos bíceps femoral (bf), semitendíneo (smt), semimembranáceo (smb), ramo do nervo cutâneo lateral da sura (cls), e a divisão em nervo fibular comum (fc) e nervo tibial (tb). (c) Mmembro pélvico direito com os músculos semitendíneo ebíceps femoral rebatidos, onde é possível observar o nervo isquiático (isq) bifurcando-se em nervo tibial (Tb), nervo fibular comum (fc), nervo fibular superficial (fs) e nervo fibular profundo (fp). Têm-se o ramo para os músculos bíceps femoral (bf) e semitendíneo (smt), e o nervo cutâneo lateral da sura (cls). 
0 nervo isquiático do mão-pelada é formado a partir de fibras ventrais oriundas do sexto e sétimo nervos lombares e do primeiro nervo sacral, disposição observada também em preá (Oliveira et al. 2010), entretanto, em gato doméstico, cão e mocó, observa-se semelhança na origem deste nervo na região lombar (sexto e sétimo nervos lombares), diferindo a formação na região sacral (primeiro e segundo nervos sacrais) (Evans \& De Lahunta 1994, Guimarães et al. 2005, Lacerda et al. 2006).

Em Cebus apella as fibras são originadas pelo quarto e quinto nervos lombares e primeiro, segundo e terceiro nervos sacrais (Carvalho-Barros 2002) e em suínos da linhagem Per Ar Lan origina-se no quinto nervo lombar e primeiro e segundo nervos sacrais (Santos 2010). Em ovinos da raça Morada Nova são formados a partir do sétimo nervo lombar e primeiro e segundo nervos sacrais (Sousa 2008). Em veados catingueiros o nervo isquiático se forma com o sexto nervo lombar e primeiro, segundo e terceiro nervos sacrais (De Camargo et al. 2008), e em fetos de bovinos azebuados as fibras partem do sexto nervo lombar e primeiro e segundo nervos sacrais (Ferraz et al. 2006). Uma síntese dos dados observados sobre a origem do nervo isquiático em mão-pelada e outras espécies, inclusive o homem, podem ser visibilizados no Quadro 1, devido ao fato de que o nervo isquiático apresenta origem semelhante entre espécimes, mesmo de ordens diferentes.

\section{Quadro 1. Ramos ventrais que participam da formação do nervo isquiático em diferentes espécies}

\begin{tabular}{lcc}
\hline Espécie & Ramos ventrais envolvidos & Referências \\
\hline Mão-pelada & L6, L7 e S1 & \\
Cães & L6, L7, S1 e S2 & Evans \& De Lahunta 1994 \\
Gatos & L6, L7, S1 e S2 & Guimarães et al. 2005 \\
Suínos & L5, S1 e S2 & Santos 2010 \\
Mocós & L6, L7, S1 e S2 & Lacerda et al. 2003 \\
Caprinos & L5, L6, S1 e S2 & Lima et al. 2008 \\
Ovinos & L7, S1, S2 e S3 & Sousa 2008 \\
Bovinos & L6, S1 e S2 & Campos et al. 2003 \\
Preás & L6, L7 e S1 & Oliveira et al. 2010 \\
Veados catingueiros & L6, S1, S2 e S3 & De Camargo et al. 2008 \\
Cebus apella & L4, L5, S1, S2 e S3 & Carvalho-Barros 2002 \\
Homem & L4, L5, S1, S2 e S3 & Gray 1988
\end{tabular}

0 trajeto do nervo isquiático no gato, conforme achados de Guimarães et al. (2005), emerge na cavidade pélvica através do forame isquiático maior, entre os músculos piriforme e glúteo profundo. No cão, assim como o mão- pelada, o nervo isquiático deixa a cavidade pélvica através do forame isquiático maior, entre os músculos glúteo superficial, bíceps femoral e abdutor caudal crural (Schwarze \& Schoder 1970, Dyce et al. 1997). Em preás o nervo isquiático deixa a cavidade pélvica através do forame isquiático maior entre os músculos glúteo médio e glúteo profundo e nos mocós inclui o músculo glúteo superficial (Santos et al. 2006, Oliveira et al. 2010).

Assim como na origem do nervo isquiático, a escassez de trabalhos científicos acerca do tema remete à comparação de sua distribuição com espécimes de outras ordens. 0 nervo distribui-se para os músculos da região glútea, observam-se ramos para o músculo glúteo profundo e piriforme no gato doméstico (Guimarães et al. 2005), ramos para os músculos glúteo médio e glúteo profundo em fetos de bovinos azebuados e em caprinos da raça Saanen (Campos et al. 2003, Lima et al. 2008), ramos para os músculos glúteo profundo e glúteo bíceps (De Camargo et al. 2008) em veados catingueiros, ramos para os músculos glúteo superficial, glúteo bíceps e glúteo médio (Santos 2010) em suínos da linhagem Per Ar Lan e ramos para o músculo glúteo bíceps, glúteo médio e glúteo profundo em caprinos (Lima et al. 2008), dados estes que corroboram com nossos achados.

Observam-se ramos do nervo isquiático em mão-pelada para os músculos gêmeos e quadrado femoral, assim como no cão, gato e suíno da linhagem Per Ar Lan (Schwarze \& Schröder 1970, Dyce et al.1997, Santos 2010) , sendo que estes não são observados em mocós e preás (Santos et al. 2006, Oliveira et al. 2010). Em fetos de bovinos azebuados, em caprinos da raça Saanen (Campos et al. 2003, Lima et al. 2008), e no veado catingueiro (De Camargo et al. 2008), observa-se ramo apenas para os músculos gêmeos e em ovinos da raça Morada Nova (Sousa 2008) não apresentam ramos para os músculos gêmeos e quadrado femoral, e sim para o músculo quadríceps femoral.

Conforme analisado em mão-pelada, observam-se ramos do nervo isquiático para os músculos semimembranáceo, semitendíneo e bíceps femoral, dados estes que corroboram com os achados para animais domésticos (Schwarze \& Schröder 1970, Dyce et al.1997), fetos de bovinos azebuados (Campos et al. 2003), mocós (Santos et al. 2006), preás (Oliveira et al. 2010), veados catingueiros (De Camargo et al. 2008), caprinos da raça Saanen (Lima et al. 2008), ovinos da raça Morada Nova (Sousa 2008) e em suínos da linhagem Per Ar Lan (Santos 2010).

0 nervo isquiático distribui-se para o músculo adutor em fetos de bovinos azebuados (Campos et al. 2003), mocós (Santos et al. 2006) e preás (Oliveira et al. 2010). Estes autores não descrevem quais músculos adutores que são supridos, sendo que no mão-pelada, observa-se um suprimento para o músculo adutor magno. Em veado catingueiro (De Camargo et al. 2008) e em caprinos da raça Saanen (Lima et al. 2008) não foram observados ramos do isquiático para o músculo adutor.

Na espécie em questão e nos demais animais analisados para comparação, observou-se que o nervo isquiático divide-se, na região medial da coxa, em dois ramos principais: o nervo tibial que supre os músculos caudais à tíbia e a fíbula e emite ramos para o joelho e o nervo fibular comum, que se divide em nervo fibular superficial e profundo, suprindo os músculos flexores do tarso e extensores dos dedos (Schwarze \& Schöder 1970, Dyce et al. 1997, Campos et al. 2003, Guimarães et al. 2005, Santos et al. 2006, De Camargo et al. 2008, Lima et al. 2008, Sousa 2008, Oliveira et al. 2010). 0 estudo desse nervo oferece contribuições para a clínica médica e cirúrgica em animais silvestres e para futuros estudos sobre a biologia geral da espécie.

\section{CONCLUSÕES}

0 nervo isquiático do mão-pelada origina-se do ramo ventral do sexto e sétimo nervos lombares e primeiro nervo sacral, assemelhando-se com a origem do preá. Em seu 
trajeto, o nervo deixa a cavidade pélvica através do forame isquiático maior, apresentando-se envolvido pelos músculos glúteo superficial, bíceps femoral e abdutor caudal crural, trajeto este semelhante com o cão.

Em mão-pelada, o nervo isquiático distribui-se para os músculos glúteo bíceps, glúteo médio, glúteo profundo, assim como observado em caprinos da raça Saanen, músculos quadrado femoral e gêmeos, assemelhando-se em cão, gato e suíno, músculos bíceps femoral, semitendíneo, semimembranáceo, observado em todas as espécimes analisadas e adutor magno, observado apenas em mão-pelada. Em todas espécimes comparadas, o nervo isquiático termina bifurcando-se em nervo tibial e fibular comum.

0 conhecimento anatômico do padrão nervoso de mão-pelada é de fundamental importância em pesquisas que se referem à distribuição do nervo isquiático, levando-se em consideração as variações anatômicas.

\section{REFERÊNCIAS}

Aversi-Ferreira T.A., Silva M.S.L.S., Pereira-de-Paula J., Gouvêa-e-Silva L.F. \& Penha-Silva N. 2005. Anatomia comparativa dos nervos do braço de Cebus apella: descrição do músculo dorsoepitroclear. Acta Sci. Biol. Sci. 27:291-296.

Aversi-Ferreira R.A.G.M.F., Marin K.A., Silva F.O.C. \& Aversi-Ferreira T.A. 2011. Comparative anatomy of the thigh nerves of Cebus libidinosus (Rylands et al., 2000). Pesq. Vet. Bras. 31:261-266.

Carvalho-Barros R.A. 2002. Estudos anatômicos dos plexos lombar, sacral e coccígeo do macaco Cebus apella: origem, composição e nervos resultantes. Dissertação de Mestrado em Anatomia dos Animais Domésticos, Faculdade de Medicina Veterinária e Zootecnia, USP, São Paulo, SP. 146p.

Câmara T. \& Murta R. 2003. Mamíferos da Serra do Cipó. PUC-Minas/Museu de Ciências Naturais, Belo Horizonte. 60p.

Campos D.B., Silva F.O.C., Severino R.S., Drummond S.S. \& Lima E.M.M., Bombonato P.P. \& Santana M.I.S. 2003. Origem e distribuição dos nervos isquiáticos em fetos de bovinos azebuados: origin and distribution of the ischiatic nerves in fetuses of the crossbred zebu cattle. Ars Vet. 19:219-223.

Carvalho C.T. 1983. Lista Nominal dos Mamíferos Brasileiros. Boletim Técnico do Instituto Florestal de São Paulo, São Paulo. 115p.

Cubas Z.S., Silva J.C.R. \& Catão-Dias J.L. 2006. Carnivora - Procyonidae (Quati, Mão-pelada, Jupará), 571p. In: Ibid. (Eds), Tratado de Animais Selvagens: medicina veterinária. Roca, São Paulo.

De Camargo V.M.F., Guerra R.R., Tranquilim M.V. \& Campos D.B. 2008. Origem e distribuição dos nervos isquiáticos no veado catingueiro (Mazama gouazoubira). Anais 35을 Conbravet, Gramado, RS. 3p. (Resumo)

Dyce K.M., Sack W.O. \& Wensing C.J.G. 1997. 0 membro anterior dos carnívoros, 252-253p. In: Ibid. (Eds), Tratado de Anatomia Veterinária. Guanabara, Rio de Janeiro.

Ellenberger W. \& Baum H. 1985. Handbuch der vergleichenden Anatomie der Haustiere. Springer, Berlin. 917p.

Emmons L.H. \& Feer F. 1997. Neotropical rainforest mammals: A field guide. University of Chicago Press, Chicago. 307p.

Evans H.E. \& De Lahunta A. 1994. Guia para Dissecção do Cão. Guanabara, Rio de Janeiro. 206p.

Ferraz R.H.S., Lopes G.R., Melo A.P.F. \& Prada I.L.S. 2006. Estudo anatômico da porção intrapélvica do nervo isquiático em fetos de bovinos azebuados. Braz. J. Vet. Res. Anim. Sci. 43:302-308.
Ghoshal N.G. 1986. Nervos espinhais, 1603-1614p. In: Getty R. (Ed.), Sisson and Grossman Anatomia dos Animais Domésticos. Editora Guanabara Koogan, Rio de Janeiro.

Guimarães G.C., Machado M.R.F., Santos A.F.Q., Vieira L.G., Souza A.G.,Silva J.M.M. \& Kaminishi A.P.S. 2005. Origem e distribuição do nervo isquiático no gato doméstico (Felis catus domesticus Linnaeus, 1758). Biosci. J. 21:189-195.

Gray H. 1979. Gray's Anatomy. Guanabara Koogan, Rio de Janeiro. 1147p.

International Committee on Veterinary Gross Anatomical Nomenclature 2005. Nomina Anatomica Veterinaria. $5^{\text {th }}$ ed. Hannover. 166p.

Júnior O.C \& Luz N. C. 2008. Pegadas Séries Boas Práticas. Edufpa, Belém. 68p.

Konig H.E. \& Liebich H.G. 2004. Anatomia dos Animais Domésticos: texto e atlas colorido. Artmed, Porto Alegre. 400p.

Lacerda P.M.O. Moura C.E.B., Miglino M.A., Oliveira M.F. \& Albuquerque J.F.G. 2006. Origem do plexo lombossacral de mocó (Kerodon rupestris). Braz. J. Vet. Res. Anim. Sci. 43:620-628.

Lima E.M.M., Silva F.O.C., Severino R.S., Drummond S.S., Campos D.B., Santana M.I.S. \& Moraes D.D.A. 2008. Origem e distribuição dos nervos isquiáticos em caprinos da raça Saanen. Ciência Rural 38:372-377.

Lima V.M., Rezende A.L., Ferreira J.R. \& Pereira K.F. 2010a. Distribution of mesenteric cranial artery in the small intestine of crab-eating raccoon (Procyon cancrivorus Cuvier, 1798). Acta Sci, Biol Sci. 32:175-179.

Lima V.M., Pereira F.C. \& Pereira K.F. 2010b. Estudo morfológico dos músculos do antebraço de mão-pelada, Procyon cancrivorus Cuvier, 1798. Biosci. J. 26:109-114.

Miranda E.E. 2003. Natureza, conservação e cultura: ensaios sobre a relação do homem com a natureza no Brasil. Metalivros, São Paulo.180p.

Morato R.G., Rodrigues F.H.G., Eizirik E., Mangini P.R. \& Azevedo F.C.C. 2004. Plano de ação: pesquisa e conservação de mamíferos do Brasil. Ibama, Brasília. 52p.

Nowak R.M. 1999. Walker's Mammals of the World. John Hopkins University Press, Baltimore. 1629p.

Oliveira G.B., Rodrigues M.N., Sousa E.S., Albuquerque J.F.G., Moura C.E.B., Ambrósio C.E., Miglino M.A. \& Oliveira M.F. 2010. Origem e distribuição dos nervos isquiático do preá. Ciência Rural 40:1741-1745.

Pereira F.C., Lima V.M. \& Pereira K.F. 2010a. Descrição anatômica dos músculos da perna de Procyon cancrivorus (Cuvier 1798). Revta Biotemas 23:159-165.

Pereira F.C., Lima V M. \& Pereira K.F. 2010b. Morfologia dos músculos da coxa de mão-pelada (Procyon cancrivorus Cuvier, 1798. Ciênc. Anim. Bras. 11:947-954

Reis N.R., Peracchi A.L., Pedro W.A. \& Lima I.P. 2006. Mamíferos do Brasil. Edição dos Autores, Londrina. 266p.

Santos R.C., Albuquerque J.F.G., Silva M.C.V., Moura C.E.B., Chagas R.S.N., Barbosa R.R. \& Miglino M.A. 2006. Anatomia do nervo isquiático em mocós (Kerodon rupestris Wied, 1820) aplicada à clínica de animais silvestres. Braz. J. Vet. Res. Anim. Sci. 43:647-653.

Santos L.A. 2010. Origem e distribuição do nervo isquiático em fetos de suínos (Sus scrofa domesticcus Linnaeus, 1758) da linhagem Per Ar Lan. Dissertação de Mestrado em Saúde Animal, Universidade Federal de Uberlândia, Uberlândia, MG. 54p.

Schwarze E. \& Schröder L. 1970. Introdución a la Anatomia Veterinária del Aparato Locomotor. Acribia, Zaragoza. 318p.

Silva F. 1994. Mamíferos silvestres. Fundação Zoobotânica do Rio Grande do Sul, Porto Alegre. 246p.

Sousa E.S. 2008. Anatomia do nervo isquiático em ovinos da raça morada nova aplicada a clínica de pequenos ruminantes. Dissertação de Mestrado em Ciência Animal. Universidade Federal Rural do Semi-árido, Mossoró, RN. 41p. 